$3 \%$ Saline (AHS) $15 \mathrm{ml} / \mathrm{kg}, \mathrm{n}=10$, over $30 \mathrm{~min}$. Hemodynamic parameters determined by femoral arterial thermodilution calibrated pulse contour analysis, central venous saturation $\left(\mathrm{SvO}_{2}\right)$, and intramucosal gastric $\mathrm{pH}(\mathrm{pHi})$ were recorded before and after fluid load. Non-parametric correlations between pre-infusion parameters and post-infusion changes with cardiac index increase $(\Delta \mathrm{CI})$ were analyzed.

Results CI (median; IOR) increased from 2.1 (1.7-2.7) to 4.1 (3.64.6) $\mathrm{L} / \mathrm{min} / \mathrm{m}^{2}$. There were no correlations between $\Delta \mathrm{CI}$ and preinfusion parameters or post-infusion changes in most parameters. Only pre-infusion stroke volume index (SVI) and global end diastolic volume index (GEDVI) showed strong negative correlation (SVI r: -0.61, p: 0.009; GEDVI r: -0.75, p: 0.001). $\Delta$ CI showed also strong correlation with SVI increase (r: 0.89, p: 0.000) and GEDVI increase ( $\mathrm{r}$ : 0.88, p: 0.000).

Conclusion Pre-infusion SVI and GEDVI were predictor parameters of fluid response in this model of hemorrhagic shock. Other parameters previously proposed as predictors of fluid response as $\mathrm{SvO}_{2}, \mathrm{PPV}$ and SVV were not able to predict changes in cardiac index.

\section{MANAGEMENT OF ACUTE CIRCULATORY FAILURE IN CHILDREN BASED ON THE EVALUATION BY ECHOCARDIOGRAPHY IN PEDIATRIC INTENSIVE CARE UNIT (PICU)}

doi:10.1136/archdischild-2012-302724.0793

K El Halimi, H Bouguetof, MA Negadi, D Boumendil, ZC Mentouri. Pediatric Intensive Care Unit, Faculty of Medicine - Oran University, Oran, Algeria

Background and Aims Echocardiography has an important role to perform in the PICU, as it is an efficient, accurate, non invasive diagnostic modality that can aid the intensivist in the management of the acute circulatory failure in children to Improve the hemodynamic Management.

The Aim is to describe how echocardiography can answer 3 of the more commonly asked questions that arise in the PICU: Complete the clinical diagnosis, guide the therapeutics, and Repeat the measures for the evaluation.

Methods In this prospective study, children who had an acute circulatory failure, the evaluation of the cardiaque output, contractility and the indications of filling were obtained by echocardiography. Each patient had a measurement before therapeutic and after to evalulate treatment.

Results In 20 children, 06 patients had septic shock, 09 with sever sepsis and 05 with severe brain injury. Median age was 3.5 years.

Intervention Standardized volume expansion (VE) when the Respiratory variations in aortic blood flow ( $\Delta$ V Peak Ao $>12 \%$ ), the VE-induced increase in LV stroke volume was $>15 \%$.

Treatment inotrope when, is an acute circulatory failure related to impaired myocardial contractility responsible for a decrease in cardiac output (Cardiogenic shock or myocardial dysfunction in the septic shock).

Norepinephrine when the mean pressure decrease with normal myocardial contractility and cardiaque output.

Conclusion Echocardiography is a incountournable tool in the evaluation and management of acute circulatory failure in children in PICU because its guide therapeutic, evalulate the efficiency of treatment and improve the surveillance.

\section{A PDA SCORE AT 48 TO 72 HOURS OF AGE (MANITOBA SCORE) PREDICTS A HEMODYNAMICALLY SIGNIFICANT PDA}

doi:10.1136/archdischild-2012-302724.0794
YN Elsayed, NICU Hemodynamic Group. Pediatrics, University of Manitoba, Winnipeg, $M B$, Canada

Objectives To determine whether a composite PDA score (Manitoba score), determined at 48-72 hours of age can predict a hemodynamically significant PDA (HSPDA) requiring closure in Infants $<31$ weeks.

Study Design Infants $<31$ weeks GA, admitted August 2010 to September 2011, to NICU Winnipeg, Canada, following parental consent, had a blinded echocardiogram and a novel PDA score determined at 48-72 hours postnatally. The PDA score is a numerical score (maximum 28) incorporating echocardiographic parameters reflective of both volume and pressure overload (max score 15), and clinical, radiological and laboratory features of both pulmonary over-circulation and systemic hypo-perfusion (max score 13). PDA diameter $>1.5 \mathrm{~mm}$ with left to right non-restrictive shunt by echo was considered for this study the reference standard for HSPDA requiring treatment. All components of the score were correlated with this reference standard.

Results 70 of 132 eligible neonates were studied. HSPDA was present in $24(34 \%)$ infants, a non significant PDA in $32(46 \%)$ and no PDA in 14 (20\%). Infants with HSPDA were of lower birth weight and less mature than those without (non-HSPDA/no PDA) ( $905 \pm 46$ vs. $1218 \pm 43$ grams; $p<0.001,28.6 \pm 0.3$ vs. $26.8 \pm 0.3$ weeks). Both the clinical and echo component correlated strongly with each other and with overall score $(p<0.001$, Kendall's tau test. The PDA score and components significantly predicted HSPDA.

Conclusion The Manitoba PDA score performed at 48-72 hours of age predicts HSPDA who eventually received treatment. Use of PDA score may reduce the number of infants who are treated with non significant PDA.

\section{ARTERIAL BLOOD PRESSURE VARIATION IN CRITICALLY ILL NEWBORNS. CAN WE PREDICT THE VOLUME STATUS?}

doi:10.1136/archdischild-2012-302724.0795

WP de Boode. Neonatology, Radboud University Nijmegen Medical Centre, Nijmegen, The Netherlands

Volume expansion is one of the most frequent used interventions in critically ill newborns, despite lack of hard evidence. In a case of a truly hypovolaemic patient, for example after massive hemorrhage secondary to an abruptio placentae, volume expansion is life-saving. However, volume expansion in a normo- and/or hypervolemic newborn infant is not without risk. Excessive fluid intake is associated with a disturbed neurologic outcome, an increased prevalence of chronic lung disease and an increased mortality. It would be profitable when the volume status of a critically ill newborn infant could objectively be assessed so the response to volume expansion could be predicted ('fluid responsiveness' - FR). The clinical assessment of the volume status is rather unreliable. Recently new dynamic parameters are introduced that reliably predict fluid responsiveness in ventilated adult patients. These variables, like 'pulse pressure variation' (PPV) and 'systolic pressure variation' (SPV), are based on arterial blood pressure variations secondary to mechanical ventilation (heart-lung interaction). This inspired us to study the phenomenon of arterial blood pressure variation in critically ill newborn infants under several clinical conditions. The (preliminary) data will be presented.

\section{PALMER'S POINT FOR SAFE INSERTION OF PERCUTANEOUS DIALYSIS CATHETER IN NEONATES}

doi:10.1136/archdischild-2012-302724.0796

B Banieghbal. Paediatric Surgery, Netcare and Life Health Care Hospital Groups, Johannesburg, South Africa 